\title{
Analyzing and correcting for contaminating magnetic fields at the Brorfelde geomagnetic observatory due to high voltage DC power lines
}

\author{
Cathrine Fox Maule ${ }^{1}$, Peter Thejll ${ }^{1}$, Anne Neska ${ }^{2}$, Jürgen Matzka ${ }^{1}$, Lars William Pedersen ${ }^{1}$, and Anna Nilsson ${ }^{1}$ \\ ${ }^{1}$ Danish Meteorological Institute, Lyngbyvej 100, 2100 Copenhagen Oe, Denmark \\ ${ }^{2}$ Institute of Geophysics Polish Academy of Sciences, Ul. Ks. Janusza 64, 01-452 Warszawa, Poland
}

(Received May 13, 2009; Revised July 20, 2009; Accepted July 21, 2009; Online published December 21, 2009)

\begin{abstract}
The magnetic field observations made at the geomagnetic observatory Brorfelde (BFE), Denmark, are subject to disturbances of several nT caused by two single wire high-voltage DC power lines connecting Scandinavia with Central Europe. We have analysed how the magnetic disturbances relate to the currents in the power lines and found a linear relationship. Using linear regression on carefully selected parts of the available data series we have determined the factors of proportionality. Based on the simple relationship between the currents in the power lines and the observed magnetic disturbances, we have derived a method to correct the observatory data for a significant part of the disturbances. In addition we have investigated the influence of the disturbances on the determination of the $K$-index of the observatory. We found that the $K$-index based on the original disturbed data compared to the $K$-indices calculated from corrected data was different in $4 \%$ of the 3 -hour intervals for the investigated period.
\end{abstract}

Key words: Geomagnetism, magnetic observatory, power lines, $K$-index.

\section{Introduction}

In 2005 a variometer station (ROE) was established on the island of Roemoe about $200 \mathrm{~km}$ west of the Danish geomagnetic observatory at Brorfelde (BFE). Comparison of data from the two stations soon revealed that one of the stations was subject to magnetic disturbances of unknown origin. It has in the past few years been established that the magnetic disturbances occur at the Brorfelde observatory, and that they are caused by two high-voltage DC power lines connecting the Scandinavian power grid with the central European one (Matzka et al., in press).

The consequences of the magnetic disturbances are that data retrieved at BFE from December 1994 and thereafter are subject to an error of up to $6 \mathrm{nT}$ in the $H$-component and up to $3.5 \mathrm{nT}$ in the $Z$-component. As the power lines are used continuously, practically all data collected since 1994 are contaminated. Occasionally both power lines may not be transferring any current for an hour or two such that the observations at BFE are undisturbed, but this is the exception rather than the rule.

The disturbances by the power lines mean that data from BFE does not properly reflect the natural variations of the magnetic field, as measurements at magnetic observatories are supposed to (e.g. Wienert, 1970; Jankowski and Sucksdorf, 1996). The field values from magnetic observatories are used for many purposes, e.g. for global field modelling, and determination of declination and magnetic indices. For these purposes data should as far as possible be devoid of contaminating magnetic fields from anthro-

Copyright (c) The Society of Geomagnetism and Earth, Planetary and Space Sciences (SGEPSS); The Seismological Society of Japan; The Volcanological Society of Japan; The Geodetic Society of Japan; The Japanese Society for Planetary Sciences; TERRAPUB pogenic sources. However, it does happen that there are disturbances in magnetic measurements at observatories e.g. from electrified railways (e.g. Pirjola et al., 2007 and references therein; Lowes, 2009 and references therein). Particularly in densely populated regions it may be difficult to find a location for an observatory, which is undisturbed, and moving observatories is a lengthy and difficult task. Therefore we have attempted to derive a method to correct the magnetic data from BFE for the disturbances caused by the power lines. We have investigated how the observed magnetic disturbances are related to the current transmitted in the power lines, with the purpose of determining their relationship. Fortunately the variations of the strengths of the currents are known quite well and there exist records of how the transmitted power has varied in the past several years. This has allowed us to develop a method for correcting past hourly mean values at BFE since October 2000, and for the present and future to correct minute data removing more than $80 \%$ of the error caused by the power lines, significantly improving the data from BFE. In addition, we have investigated how the magnetic disturbances of the power cables influence the determination of the $K$-index values of the observatory.

\section{Kontek and Baltic Cable}

The two power lines responsible for the magnetic disturbances at BFE are Baltic Cable, operated by Baltic Cable $\mathrm{AB}$, and Kontek operated by Energinet.dk. Both cables are high-voltage DC power lines, but differ from most other DC power lines in that they are single-wire power lines. Often DC power lines consist of two parallel wires a few meters apart carrying the current and the return current respectively. Such a configuration is favourable for the ge- 


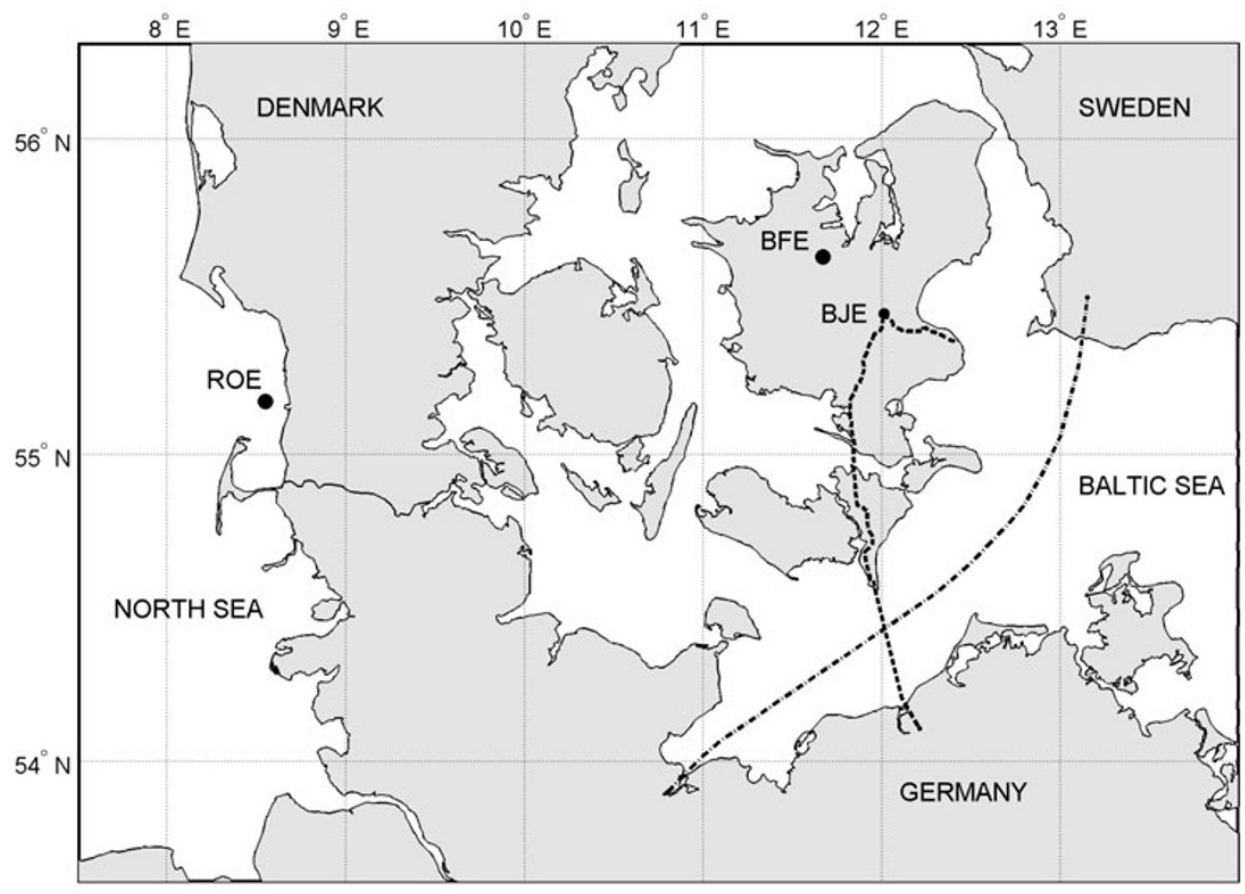

Fig. 1. Map of the region showing the locations of Brorfelde observatory (BFE) and Roemoe variometer station (ROE), as well as of the Kontek converter station at Bjaeverskov (BJE). Baltic Cable is shown with the dash-dot line and Kontek with the dashed line. At its closest point, the Kontek cable is at a distance of $29 \mathrm{~km}$ from the observatory BFE.

omagnetic community, because in this case the magnetic field from the two wires cancel each other to a large extent and therefore magnetic disturbances are limited. However, this cancellation does not occur for Kontek and Baltic Cable, because only one wire is leading the current, the return current is going through the Baltic sea and the ground and therefore these power lines cause disturbing fields at BFE.

Kontek, which runs from Bjaeverskov in Denmark to Bentwisch in Germany (Fig. 1), has been in service since 1996; it can carry a current of $1500 \mathrm{~A}$ and has a voltage of $400 \mathrm{kV}$. Baltic Cable, which runs from Trelleborg in southern Sweden to Lübeck-Herrenwyk in Germany, was taken into service in December 1994; it typically has a voltage of $450 \mathrm{kV}$ and can carry a current of up to $1335 \mathrm{~A}$. Power can be transported in either direction; however, the technical specifications of the power lines are such that the current always runs in the same direction. In the Kontek cable, the current always runs from Bentwisch to Bjaeverskov (from south to north), and from Bjaeverskov to the coast at Stevns (from west to east), and in the Baltic cable the current always runs from Lübeck-Herrenwyk to Trelleborg (from south-west to north-east). The return currents are for both cables led through the Baltic Sea (and the west-east link from Bjaeverskov to the coast), with the cathode on the German side.

The currents in the two cables are changed regularly following the prices of electricity on the European market. As power is sold on an hour-to-hour basis at the European power exchange, Nordpool, the currents in the power lines are typically changed at the beginning of an hour and kept constant for the rest of that hour. When the direction of power transport is changed the current momentarily becomes zero.
Change in power in Baltic Cable, which is computer controlled, occurs at the beginning of the hour with a constant rate of change of $30 \mathrm{MW} /$ minute and is kept constant until the next hour. Out of these constraints it is possible to reconstruct minute values of Baltic Cable power from the hourly mean power values published on www.nordpool.com. Values of current, which unfortunately are not available to us, would have been preferable as magnetic disturbances are directly proportional to the current and not to the power. However, the voltage is usually kept at a level close to $450 \mathrm{kV}$, and hence for our purpose we assume that the current is proportional to the power.

Changes in Kontek current often occur several minutes before or after the change of the hour. Therefore minute data from Kontek are necessary in order to analyse the effects of changes in the Kontek current on the magnetic field measurements at BFE. As of mid-November 2008 we have received nearly continuous on-line real time current data from the Kontek cable.

To get an idea of the order of magnitude of the magnetic field, that the cables (without return currents) cause at BFE, the magnetic field of an infinite wire at the minimum distance between BFE and each cable can be calculated. For Kontek, at a mimimum distance of $29 \mathrm{~km}$, a current of 1000 A would cause a field of $7 \mathrm{nT}$. For Baltic, using a minimum distance of $80 \mathrm{~km}$, a similar current would cause a field of $2.5 \mathrm{nT}$. Both cables and BFE are lying close to sea level, so the field from the cables will predominantly affect the vertical component of the magnetic field at BFE, whereas the horizontal components will be virtually unaffected. 


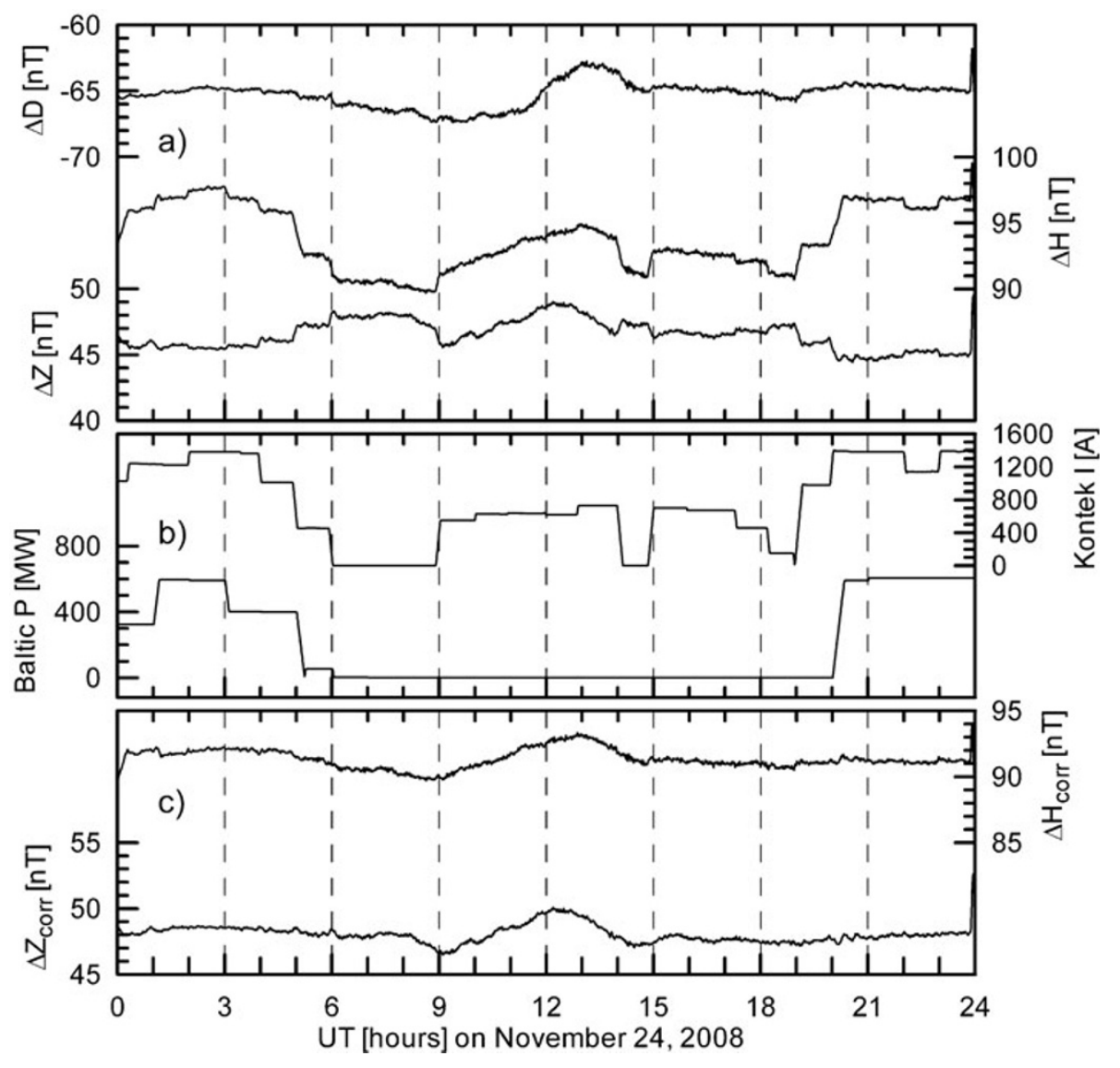

Fig. 2. Magnetic field values and current/power values from November 24th, 2008; all are 1-minute values. (a) The difference between the magnetic field measurements at $\mathrm{BFE}$ and ROE for the $D$-, $H$ - and $Z$-component. It is clear that unnatural jumps occur several times in the $H$ - and $Z$-components at the change of the hour. (b) The current in Kontek and the power transmitted by Baltic Cable the same day. The connection between the jumps in the magnetic field differences and the changes of current/power is clear. (c) The corrected values of the difference between BFE and ROE for the $H$ and Z-component, using the FPs from the Nov 08-Jan 09 period for the correction. It is clear that the corrections strongly reduce jumps and offsets caused by the power lines.

\section{Data Periods}

We have analysed data from four different periods where the required data have been available. From January 1st to March 9th 2007 and again from February 22nd to April 18th 2008 Kontek was temporarily shut down for maintenance, making these two periods ideal for studying the effect of Baltic Cable alone. In order to analyse the effects of Kontek, minute data are necessary; these have been available to us from two periods. Energinet.dk kindly supplied data from October 1st to October 20th 2007 and since midNovember 2008 we have in cooperation with Energinet.dk collected $1 \mathrm{~Hz}$ Kontek current data with precise time stamp and we subsequently average these data to minute mean values in the same way as the magnetic data are averaged. At both $\mathrm{BFE}$ and ROE the magnetic field components $H, Z$ and $D$ are measured with a $1 \mathrm{~Hz}$ sampling, from which minute values are obtained by taking the mean value over a 60 -second period. For our analysis we use 1-minute mean values of power, current and magnetic field all of which are centred on the half minutes.

\section{Least Squares Analysis}

The natural variations in the magnetic field at BFE are generally much larger than the contribution from the power lines. To better isolate the contribution from the power lines, we eliminate much of the contribution from natural current systems by taking the difference between the observed fields at BFE and ROE (subtracting ROE values from BFE) and compare this with the power line data. At both places the variation of the horizontal $(H)$ and the vertical $(Z)$ components are measured as well as the variation of the declination $(D)$, which here is expressed as the variation of the horizontal east component measured in nT. The $H-, D-$ and $Z$-differences are shown in Fig. 2(a) along with the current/power from the two cables from November 24th, 2008 in Fig. 2(b). It is clear that when the current in either of the power lines is increased $\Delta H$ increases and $\Delta Z$ decreases, and when the current is decreased $\Delta H$ decreases and $\Delta Z$ increases. There is no apparent change in $D$.

Our initial studies showed that, to a first approximation, the magnetic disturbances caused by the power cable circuits are proportional to the current/power in the cables. Thus we postulate that the observed difference, $\Delta H$, can be described by

$$
\Delta H=\alpha_{0}+\alpha \cdot I+\beta \cdot P+r
$$

where $I$ is the current in Kontek, $P$ is the magnitude of the power transported in Baltic, $\alpha$ and $\beta$ are the factors of proportionality (FP) for Kontek and Baltic respectively, $\alpha_{0}$ an 
intercept and $r$ the residual term, which contains the difference between the natural magnetic variations at BFE and ROE, as well as measurement noise. Due to the difference in longitude of BFE and ROE a daily variation is present in the difference signal, also embedded in the residual term. There are core and crustal field differences between BFE and ROE also, but we assume they are constant over the short time intervals considered here, and do not take them into account. We use arbitrary offsets for BFE and ROE data before calculating their difference. The expressions for the other magnetic components are equivalent to Eq. (1).

To determine the FPs of the regression problem in Eq. (1), we apply the least squares method. For the least squares method to work, stationarity of the residuals is required, which the residuals in Eq. (1) are not. The consequences of non-stationarity may be that formal error estimates based on the residuals are unrealistically small, orin the most complex cases - that estimates of the coefficients themselves in Eq. (1) are biased (as well as their uncertainties). It is beyond the scope of the present paper to go into the most advanced of these topics, so instead we shall apply the best-known and simplest technique of socalled pre-whitening of all time series (e.g. Fuenzalida and Rosenblüth, 1990) in pursuit of stationarity. Pre-whitening is done by taking differences between neighbouring values in the data series, i.e. for points $i$ and $(i+1)$ in the data series. Following Eq. (1) we have

$$
\Delta H_{i}=\alpha_{0}+\alpha \cdot I_{i}+\beta \cdot P_{i}+r_{i}
$$

and

$$
\Delta H_{i+1}=\alpha_{0}+\alpha \cdot I_{i+1}+\beta \cdot P_{i+1}+r_{i+1} .
$$

Taking the difference between Eqs. (3) and (2) gives

$\left(\Delta H_{i+1}-\Delta H_{i}\right)=\alpha\left(I_{i+1}-I_{i}\right)+\beta\left(P_{i+1}-P_{i}\right)+\left(r_{i+1}-r_{i}\right)$.

This technique ensures that the coefficients $(\alpha$ and $\beta$ ) are the same as in the un-pre-whitened series (Eq. (2)), and that the residuals of the pre-whitened series in Eq. (4), $\left(r_{i+1}-r_{i}\right)$, are more likely to be independent than the residuals of Eq. (2), $\left(r_{i}\right)$. A measure of the dependency is the decorrelation length. If the data points in the series are completely uncorrelated the decorrelation length is zero, but if serial correlation is present the decorrelation length will be larger. Under the assumption that the series are well described as an auto-regressive process of order 1, the decorrelation length can be determined using the simple formula

$$
\tau=\frac{1+\mathrm{AC}_{1}}{1-\mathrm{AC}_{1}}
$$

where $\mathrm{AC}_{1}$ is the autocorrelation at lag 1 ; this follows von Storch and Zwiers (1999). We find that the decorrelation length of the residual prior to the pre-whitening is about 150 data steps (varies between components and periods), and after pre-whitening only 1-3 data steps; thus the prewhitening significantly reduces structure in the residual.

However, even after the pre-whitening the residual is not quite stationary. The difference signal between BFE and ROE is highly variable following the level of magnetic activity, which means that the standard deviation of the prewhitened residual varies with the level of magnetic activity. A second step in pursuit of stationarity is to only use data from quiet times, which we define as periods where the $K$ index of BFE is zero. As discussed later, the assignment of the $K$-index is influenced by the power cable disturbances, but the disturbances will predominantly cause the $K$-index to be assigned a higher value than it should. So when selecting periods with a $K$-index of zero most of the periods selected will have a true $K$-index value of zero. Selecting quiet days reduces the amount of data used for analysis. For the four periods in question between $10 \%$ and $25 \%$ of the data are retained for analysis, consisting of fragments of the original data series.

To solve for the factors of proportionality (FP) between the observed disturbance and the current/power we use least squares on Eq. (4). Because the current (or power) in the power lines are constant much of the time, the series of differenced current, $\left(I_{i+1}-I_{i}\right)$, and differenced power will contain a large proportion of zeros. Including a large proportion of data points representating the same value of the regressor can in principle force the least squares analysis very strongly by over-representing one point. However, we have verified that this is not a problem in the present case and therefore retain all the differenced data from quiet times for analysis.

To estimate the uncertainty of the resulting FPs we apply bootstrapping (e.g. Ebisuzaki, 1997), with replacement, on data series constructed by concatenating the selected data for each data period. Bootstrapping is a statistical method to estimate the standard deviation of the result of the least squares analysis, which is more robust towards remaining auto-correlation in the residual, than the standard deviation estimates given by the least-squares calculation. Bootstrapping is applied the following way: If a given concatenated data series has $N$ points, a set of $N$ random numbers is drawn (with replacement) from the integers 1 to $N$. Then the original data are reordered into a new data series following the order of the random numbers; here some of the original data points are present several times, while others will not be present at all. Then we solve for the FPs on the reordered data series and obtain a slightly different result than the FPs from the original data series. This procedure is repeated many times giving a distribution of the FP results. The standard deviation of this distribution estimates the standard deviation of the FP determined from the original concatenated data. Because the data series of differenced current, differenced power and differenced residual, have decorrelation lengths above zero, we need to apply block bootstrapping. In this situation, instead of making a series of $N$ random numbers, $N / L$ random numbers is drawn (with replacement), where $L$ is the block length, which is determined from the autocorrelation spectrum of the original concatenated data series. In this case the reordered data series is constructed of $N / L$ blocks of the original concatenated data series each of length $L$ in the order of the random numbers. Each random number gives the data point where a block begins and the block consists of the following $L$ data points. Using this method the reordered data series has the same decorrelation length as the original. To 
Table 1. The least squares results for the factors of proportionality and their standard deviations provided by bootstrapping for each magnetic component and data period for Baltic Cable. $N$ is the number of 1-minute data points from each period fulfilling the data selection criteria.

\begin{tabular}{lccrr}
\hline Period & $H(\mathrm{pT} / \mathrm{MW})$ & $Z(\mathrm{pT} / \mathrm{MW})$ & $D(\mathrm{pT} / \mathrm{MW})$ & \multicolumn{1}{c}{$N$} \\
\hline Jan-Mar 07 & $4.04 \pm 0.10$ & $-1.33 \pm 0.08$ & $0.08 \pm 0.23$ & 19080 \\
Oct 07 & $4.25 \pm 0.23$ & $-1.34 \pm 0.18$ & $-0.06 \pm 0.44$ & 7020 \\
Feb-Apr 08 & $3.49 \pm 0.29$ & $-1.15 \pm 0.21$ & $-0.29 \pm 0.23$ & 7740 \\
Nov 08-Jan 09 & $3.97 \pm 0.12$ & $-1.32 \pm 0.10$ & $-0.08 \pm 0.10$ & 17708 \\
\hline
\end{tabular}

Table 2. The least squares results for the factors of proportionality and their standard deviations provided by bootstrapping for each magnetic component and data period for Kontek. $N$ is the number of 1-minute data points from each period fulfilling the data selection criteria.

\begin{tabular}{lcccr}
\hline Period & $H(\mathrm{pT} / \mathrm{A})$ & $Z(\mathrm{pT} / \mathrm{A})$ & $D(\mathrm{pT} / \mathrm{A})$ & $N$ \\
\hline Oct 07 & $2.08 \pm 0.33$ & $-1.44 \pm 0.22$ & $0.18 \pm 0.18$ & 7020 \\
Nov 08-Jan 09 & $2.32 \pm 0.04$ & $-1.68 \pm 0.03$ & $0.64 \pm 0.03$ & 17708 \\
\hline
\end{tabular}

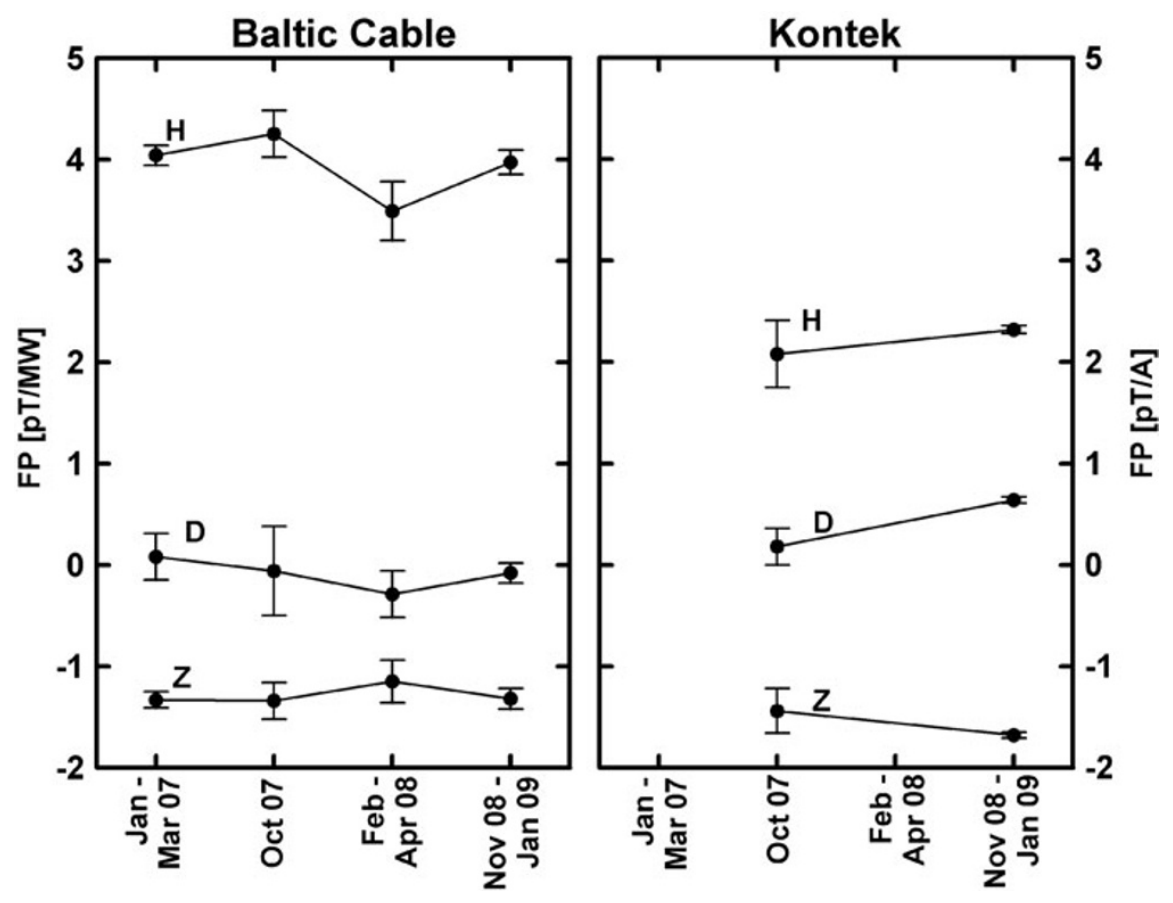

Fig. 3. The factors of proportionality for each cable for each component for the four periods with their standard deviations. The visual display shows the scatter of the FPs for the $H$-component for Baltic Cable. It is also clear that the FPs for the $D$-component are scattered around zero and in most cases zero is within the standard deviation.

determine the block length we calculated the decorrelation length for the concatenated series of pre-whitened power and current data and found it to vary between 6 and 14 data steps for the different data periods. We therefore use a block length of 14; for the four periods of data this gives between 501 and 1362 blocks. We bootstrap 10,000 times as testing showed that at this number the bootstrapping results are reproducible.

\section{Results of Least Squares Analysis}

Using the above method we have solved for the proportionality factors for Baltic Cable and Kontek. We have applied the method on all three components of the magnetic field and found consistent results for the $H$ - and $Z$ components, whereas the $D$-component appears to be unaffected by the power lines. The results for all three components for the different data periods are listed in Table 1 for Baltic Cable and Table 2 for Kontek and displayed graphi- cally in Fig. 3.

In the case of Baltic cable we observe that out of 12 determinations of coefficients, one case is about 2 standard deviations outside the average value for that componentthis is the Feb-Apr 08 value for $H$. In the $D$ and $Z$ cases smaller excursions are observed. The relatively large excursion of the FP value for $H$ based on Feb-Apr 08 data raises the question of whether it is likely that the 2 standard deviation difference occurred by chance. The probability that a normal variate is 2 standard deviations from the mean value is about $4.6 \%$. The probability of observing one such event in 12 trials is about $48 \%$. Therefore the results do not discard the hypothesis that the FPs-field component by component-are constant in time.

Overall we find that for every megawatt (about 2.2 A) that the power in Baltic is increased, there is an increase in $H$ at BFE of about $0.0040 \mathrm{nT}$, and a decrease in $Z$ of about $0.0013 \mathrm{nT}$. For every ampere the current in Kontek is in- 
creased, there is an increase in $H$ of about $0.0022 \mathrm{nT}$ and a decrease of $Z$ of about $0.0016 \mathrm{nT}$. The level of uncertainty of these average FPs is about $20 \%$ based on the variation of the FPs and their standard deviations. When both cables carry their maximum current the total change at BFE is about $+6 \mathrm{nT}$ in $H$ and about $-3 \mathrm{nT}$ in $Z$; in this case the $20 \%$ uncertainty means the error is $1.2 \mathrm{nT}$ on the $H$ component, and $0.6 \mathrm{nT}$ on the $Z$-component. The average correction on minute values in January 2009 was $+3.8 \mathrm{nT}$ on $H$ and $-2.3 \mathrm{nT}$ on $Z$. Of the entire month consisting of 44,640 minutes only data from 110 minutes were undisturbed by the power lines (no currents running). The power transfers in January 2009 were ordinary compared to other months suggesting that these levels of disturbances are typical.

\section{Discussion}

As the FPs in principle could vary in time it is interesting to speculate on possible causes of such variation. One possible cause of variation of the FPs could be regional scale changes in the conductive environment, as e.g. in the conductivity of the water or of the ground beneath BFE. In this case we would expect the FPs of the two cables to vary in the same manner, i.e. either both increase or decrease during a given period although not necessarily with the same proportion. To test this we calculated both FPs of each magnetic component simultaneously for subsets of data from the Nov 08-Jan 09 data period, where both cables were active. We found no correlation of the FPs variation, therefore we conclude that regional scale changes in the conductivity is not a cause of the variation. Another possible cause of variation in the FPs is relatively local changes in the conductive environment, i.e. the salinity of the water, close to the electrodes of the cables. One way to investigate this could be to compare the salinity of the water near the electrodes with the FPs e.g. by calculating the FPs for periods with (nearly) constant salinity. Such a study was outside the scope of this work, so we cannot rule out that there is such an effect.

The power lines contributions to the magnetic field at Brorfelde comes in part from the power lines themselves, which based on pure geometry (power lines and observatory are close to sea level) are expected to affect the $Z$ component, whereas neither the $D$ - nor the $H$-component should be affected. The rest of the contribution comes from the return current which may be running in the saline water and in the ground, and probably does both in a complex pattern determined by the land-sea distribution and other geologic conditions; part of it may run very deeply into the ground (e.g. Pirjola et al., 2007). The return current probably spreads out over a large area, and some of it may even be running in the ground beneath Brorfelde, where magnetotelluric measurements verified an east-west electric field varying with the power in both cables (Matzka et al., in press). Thus the cables with return currents form complex current loops in many directions, which make it very difficult to make a theoretical model of the problem.

However, calculating the magnetic fields at Brorfelde from the cables themselves is simple, and gives an idea of the importance of the contribution from the cable vs. the contribution from the return currents. Using the exact lo- cation of the Kontek cable, we calculated the contribution to the magnetic field at BFE from each small section of the cable and summed them up to find the total contribution. We found that the change in $Z$ at BFE due to the current in the Kontek cable is $-0.0022 \mathrm{nT} / \mathrm{A}$, which is a somewhat stronger contribution than the experimentally determined FP of $-0.0016 \mathrm{nT} / \mathrm{A}$; the difference is due to the return current. Thus we can conclude that the contribution from the return current $(0.0006 \mathrm{nT} / \mathrm{A})$ is about $1 / 3$ of the amplitude of the contribution from the cable to the disturbances on the $Z$-component. We made a similar calculation for Baltic Cable based on an apparent location of the cable, and found that the current in the cable contributes to a change in $Z$ of about $-0.0032 \mathrm{nT} / \mathrm{MW}$, again as expected a larger amplitude than the experimentally determined value of $-0.0014 \mathrm{nT} / \mathrm{MW}$. Thus for Baltic we find that the contribution from the return current is $0.0018 \mathrm{nT} / \mathrm{MW}$, more than half of the contribution from the current in the cable to the disturbance in the $Z$-component. It seems reasonable that the return current has a relatively larger contribution to the disturbances of the $Z$-component for Baltic Cable than for Kontek, as the return current for Kontek probably on average runs at a larger distance from BFE than the cable itself, whereas the opposite probably is the case with Baltic Cable. For both cables we expect the disturbance on the $H$ component to be dominated by the return currents; as the cables both lie close to sea level the field from them at BFE, which is also close to sea level, should be almost entirely vertical.

\section{Frequency-domain Method}

An alternative independent way to estimate the FPs is taking a frequency-domain based approach. This analysis technique is used for transfer function estimation in magnetotellurics and does not require pre-selection of data based on $K$-index. We have applied this method to a data fragment from October 5th to 17th, 2007. In this method the magnetic field measured in BFE $\left(H^{\mathrm{BFE}}, D^{\mathrm{BFE}}, Z^{\mathrm{BFE}}\right)^{T}$ is explained by the horizontal magnetic field in ROE

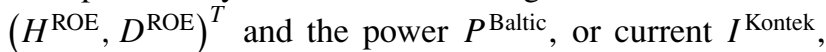
respectively, of both cables. Having transformed all these time series into frequency domain, we can write

$$
\begin{aligned}
\left(\begin{array}{c}
H^{\mathrm{BFE}} \\
D^{\mathrm{BFE}} \\
Z^{\mathrm{BFE}}
\end{array}\right)= & \left(\begin{array}{llll}
T_{H H} & T_{H D} & T_{H \text { Kontek }} & T_{H \text { Baltic }} \\
T_{D H} & T_{D D} & T_{D \text { Kontek }} & T_{D \text { Baltic }} \\
T_{Z H} & T_{Z D} & T_{Z \text { Kontek }} & T_{Z \text { Baltic }}
\end{array}\right)\left(\begin{array}{c}
H^{\mathrm{ROE}} \\
D^{\mathrm{ROE}} \\
I^{\text {Kontek }} \\
P^{\text {Baltic }}
\end{array}\right) \\
& + \text { residual. }
\end{aligned}
$$

The tensor elements $T_{i j}, i=\{H, D, Z\}, j=$ $\{H, D$, Kontek, Baltic $\}$ contain the desired transfer functions. They are estimated by minimizing the residual via least squares, details can be found in Neska (2006). The $T_{i j}$ are complex functions of the period that are displayed in form of magnitude and phase. The two left-hand side tensor columns cover the difference in the natural magnetic variations between BFE and the reference ROE, which is caused by electromagnetic induction in the laterally inhomogeneous subsurface. This kind of transfer function is relatively common in magnetotellurics (e.g. Schmucker, 

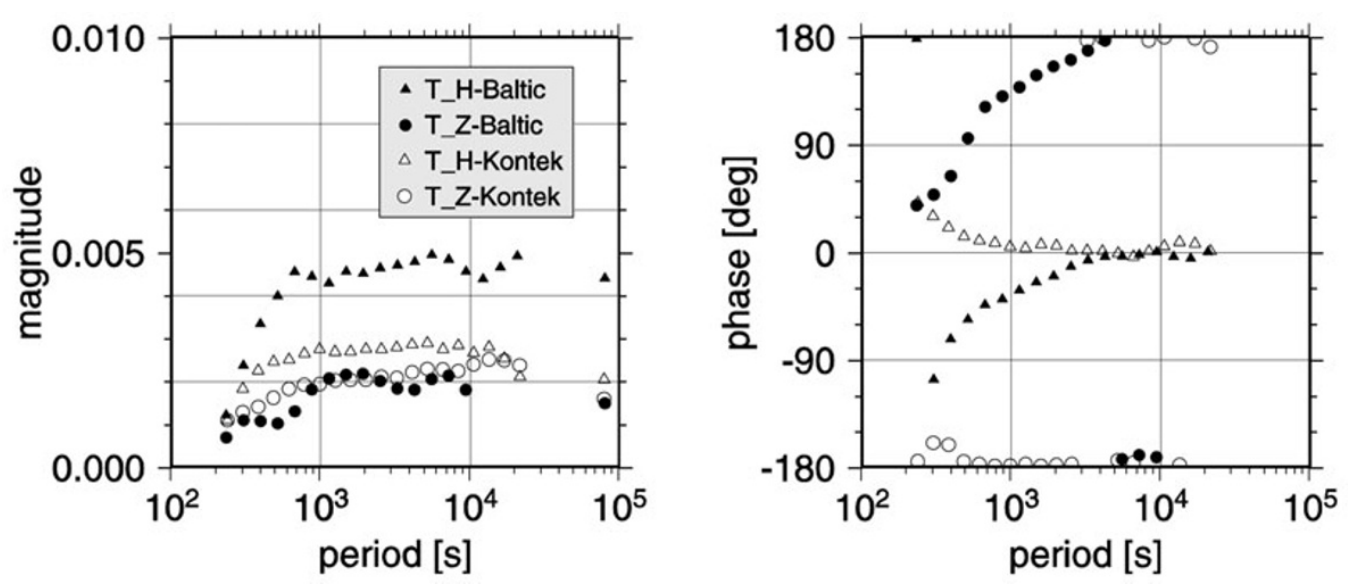

Fig. 4. The transfer functions converting the changes of cable power (or current, respectively) into the corresponding magnetic field variation measured at Brorfelde. The unit of the magnitude is nT/MW for the Baltic and nT/A for the Kontek components. For long periods, the magnitudes reflect the factors of proportionality obtained by the time-domain method, which are added at 80,000 s to the magnitude graphics. The $\pm 180^{\circ}$ phases of the $Z$ components are consistent with a negative sign. For short periods, magnitude values are reduced and significant phase-shifts occur.

1970; Soyer and Brasse, 2001; Varentsov and EMTESZ WG, 2005). In contrast, both columns on the right-hand side of the tensor describe the effect of changes in the cable time series on the BFE magnetic field, i.e. they are the counterparts to the FPs of the time-domain method. If this relationship in time-domain was described completely by a single-valued proportionality factor, then the phases of these transfer functions would be $0^{\circ}$ or $\pm 180^{\circ}$ (the latter if the sign is negative) over all periods and the magnitude would be some constant equal to that FP. According to the results presented in Fig. 4, this is the case only with periods longer than $600 \mathrm{~s}$ (Kontek) or even $4000 \mathrm{~s}$ (Baltic). For shorter periods, the magnitudes are lower and the phase differs from the long-period value in a systematic way. This phase shift can be explained partly by lacking synchronicity between BFE and cable data. The time stamp of some of the cable data from this particular period is systematically wrong by a few minutes, which also is evident from direct comparison of the time series in the time-domain. Furthermore, since the varying electromagnetic field emitted by the cables propagates through the conductive subsurface, it is subjected to electromagnetic induction, which has a frequency-dependent attenuating and phase-shifting effect and obviously concerns the shorter periods of the cable transfer functions. So the deviations from the constant proportionality assumption made in the time-domain method are not completely unexpected, and their neglect can theoretically lead to underestimated FPs. Nevertheless, the transfer function values for longer periods are in rough agreement with the FPs derived with the time-domain method, i.e. $T_{H \text { Baltic }}=0.0044 \mathrm{nT} / \mathrm{MW}$, $T_{Z \text { Baltic }}=-0.0017 \mathrm{nT} / \mathrm{MW}, T_{H \text { Kontek }}=0.0027 \mathrm{nT} / \mathrm{A}$, $T_{Z \text { Kontek }}=-0.0019 \mathrm{nT} / \mathrm{A}$, whereas the $T_{D}$ components for both cables have negligible values.

Theoretically the frequency domain method offers two possibilities to correct our time series for cable effects. First, multiplication of the left-hand tensor columns with $(H, D)^{T}$ of a mostly noise-free reference like ROE reconstructs undisturbed data, see Oettinger et al. (2001) and Neska (2006) for detailed description and application of this method. Second, multiplying the right-hand tensor columns with $\left(I^{\text {Kontek }}, P^{\text {Baltic }}\right)^{T}$ leads to the cable-generated part of magnetic variations at BFE which can be removed by subtraction from the original data. However, tests revealed that these results are problematic at very long periods that exceed the range covered by the transfer functions. Therefore data correction should be done with the time-domain method.

\section{Correcting the Observed Field}

The magnetic field observations at BFE can be corrected for a significant part of the contributions from the power lines using the FPs when the current/power transfer in the cables is known. Except for part of October 2007, we only have hourly mean values of Kontek power prior to January 2009. Therefore regarding past values from BFE we can only correct hourly mean values and not minute values. Fortunately hourly means of the power values and of the magnetic field values at BFE are obtained the same way by simple averaging over all the minute values of the hour. Therefore the linear relationship used for correcting minute values can be directly transferred to the hourly mean values as only linear processes are involved. Past hourly mean values of the power transmitted through Baltic Cable and Kontek are available from Nordpool, but only back until October 2000 for Kontek, and January 1998 for Baltic Cable. This means that BFE data from December 1994, when Baltic Cable was taken into service, until October 2000, cannot be corrected unless new archives of old power data should appear.

Since January 2009 we have had a nearly steady record of $1 \mathrm{~Hz}$ data of the Kontek current. From these we can calculate minute values, which make it possible to correct minute values of the magnetic field at BFE. An example of the correction is shown in Fig. 2(c) where the corrected difference data from November 24th 2008 using the FPs from the Nov 08-Dec 09 period are displayed. It is clear from the figure that the correction effectively removes jumps and offsets caused by the power lines, and produces difference curves that reflect the natural difference in the magnetic field be- 
tween the two sites. The correction that is applied to the BFE-ROE difference data can also be used to correct the actual BFE data. The cables also affect the measurements in ROE, but the larger distance to ROE from the cables significantly reduces the magnitude of the contaminating fields here. Therefore the correction to the actual BFE data is close to the correction of the difference data.

If the FPs are time-variable it could be worth considering whether correction of BFE data in the future should be done on limited time periods, as this would account for the variability in the FPs and potentially decrease the error by increasing the accuracy of the correction. For example the FPs could be calculated for set periods, and then the obtained FPs could be used to correct data from each period. One difficulty with this approach is setting the length of the time interval. It should be short enough that the FPs do not vary significantly within the period, but it must be long enough to ensure that there will be a sufficient amount of usable data to determine the FPs after the strongly reducing data selection is done; particularly at times of high solar activity, the data reduction will be very strong. How short the period may be is hard to say, but it is likely that no ideal set time interval exists. In addition such an approach would mean that the (final) correction of the BFE data only could be done after the time interval had ended, and not real-time. It would, however, still be possible to produce preliminary real-time corrected values based on average FP values.

\section{9. $\quad$ Effect on $K$-index}

The $K$-index (Bartels, 1957a, b; Mayaud, 1980), which reflects the magnetic activity at an observatory and the scaling of which is observatory dependent, is partly determined by variations in the $H$-component. Thus variations of the $H$-component due to changes in the currents in the power lines may cause a 3-hour interval to get an erroneous $K$ index assigned. To investigate if this occurs, we corrected the magnetic field observations from BFE from December 2008 to February 2009 for Baltic Cable effects and for Kontek effects, to the extent Kontek minute data were available. Using the automated method of Nowozynski et al. (1991) we then calculated the $K$-index for both the corrected and the original, disturbed magnetic field data and compared the results. The 90-day period has a total of 720 3-hour intervals, of these the $K$-index was different in 28 cases. In one case the $K$-index was reduced from 2 for the original data to 1 for the corrected, and in 26 cases the $K$-index was reduced from 1 to 0 . Only in one case was the $K$-index increased by correction, from 0 to 1 . Thus whereas in the original, disturbed data 266 of the 3 -hour intervals had a $K$-index of zero, this increased to 292 for the corrected data. As observed we would expect to find the erroneous $K$-values predominantly to be low $K$-values, as the interval of variation of $H$ that determines the $K$-value increases with increasing $K$-value. The maximum change in $H$ caused by the power cables is about $5.75 \mathrm{nT}$; the more disturbed the magnetic field is the less likely it is that such a change would exceed the $K$-index interval.

The larger the variation of the correction of $H$ is within a given 3-hour interval, the more likely it is that the $K$ index has been assigned an erroneous value, but even a relatively small change in the $H$-correction over a 3-hour interval of just $1 \mathrm{nT}$ can cause the $K$-index value to be shifted. In conclusion we find the effect of the power lines to influence the assignment of $K$-values at BFE, and for the investigated period the $K$-index was wrong in $4 \%$ of the cases. The $K$-index based on the original, disturbed data was mainly too high. As BFE is one of 13 selected observatories, whose $K$-index is included in the calculation of the planetary $K_{\mathrm{p}}$-index (e.g. Menvielle and Berthelier, 1991 ), also the $K_{\mathrm{p}}$-index may be influenced by the effects of the power lines. Therefore the $K$-index of BFE should preferentially be calculated on corrected data.

\section{Conclusions}

We have analysed the magnetic disturbances at the observatory in Brorfelde caused by the two high-voltage DC power lines, Kontek and Baltic Cable. The disturbances are proportional to the current in the power lines to a good approximation. We could not discard the hypothesis that the FPs are constant in time. We have determined the factors of proportionality using a least-squares method for four different data periods, and find that the $H$-component is increased by about $0.0040 \mathrm{nT}$ per megawatt of increased power in Baltic Cable and about 0.0022 nT per ampere of increased current in Kontek. The Z-component is decreased with about $0.0013 \mathrm{nT}$ for every megawatt the power in Baltic is increased, and with about $0.0016 \mathrm{nT}$ for every ampere the current in Kontek is increased. We found results in agreement with this using an independent frequencydomain based method. Using the factors of proportionality we can correct the magnetic data at BFE for much of the influence of the power lines, in the extent the current/power in the cables are known. For the period October 2000 to December 2008 we can correct the hourly mean values from Brorfelde, from January 2009 and forward we can correct minute values as well.

Finally, we have investigated the effect of the disturbances of the power cables on the assignment of the $K$ index, and find that the power cables influence the values of the observatory's $K$-index, which again may influence the determination of the $K_{\mathrm{p}}$-index.

Acknowledgments. We thank Energinet.dk for their cooperation, providing current data and exact location of the Kontek cable; in particular we thank Henrik Roland for his help. We also want to thank Nordpool for making past power data available to us. Finally we thank colleagues from other observatories who have engaged in useful discussions of this problem with us.

\section{References}

Bartels, J., The technique of scaling indices $\mathrm{K}$ and $\mathrm{Q}$ of geomagnetic activity, Ann. Intern. Geophys., Year 4, 215-226, 1957a.

Bartels, J., The geomagnetic measures for the time-variations of solar corpuscular radiation, described for use in correlation studies in other geophysical fields, Ann. Intern. Geophys., Year 4, 227-236, 1957b.

Ebisuzaki, W., A method to estimate the statistical significance of a correlation when the data are serially correlated, J. Clim., 10(9), 2147-2153, 1997.

Fuenzalida, H. and B. Rosenblüth, Prewhitening of climatological time series, J. Clim., 3(3), 382-393, 1990.

Jankowski, J. and C. Sucksdorf, Guide for magnetic measurements and observatory practice, 235 pp, IAGA, 1996.

Lowes, F. J., DC railways and the magnetic fields they produce-the geomagnetic context, Earth Planets Space, 61, i-xv, 2009. 
Matzka, J., L. W. Pedersen, C. Fox Maule, A. Neska, J. Reda, A. Nilsson, and H.-J. Linthe, The effect of high-voltage DC power lines on the geomagnetic measurements at BFE, Proceedings of XIII IAGA Workshop on Geomagnetic Observatory Instruments, Data Acquisition and Processing, in press.

Mayaud, P. N., Derivation, meaning, and use of geomagnetic indices, in Geophys. Monogr. Ser., 22, American Geophysical Union, Washington DC, 1980.

Menvielle, M. and A. Berthelier, The K-derived planetary indices: description and availability, Rev. Geophys., 29(3), 415-432, 1991.

Neska, A., Remote Reference versus Signal-Noise Separation: A leastsquare based comparison between magnetotelluric processing techniques, PhD thesis, Freie Universitt Berlin, Fachbereich Geowissenschaften, available at http://www.diss.fu-berlin.de/2006/349, 2006.

Nowozynski, K., T. Ernst, and J. Jankowski, Adaptive smoothing method for computer derivation of K-indices, Geophys. J. Int., 104, 85-93, 1991.

Oettinger, G., V. Haak, and J. C. Larsen, Noise reduction in magnetotelluric time-series with a new signal-noise separation method and its application to a field experiment in the Saxonian Granit Massif, Geophys. J. Int., 146, 659-669, 2001.

Pirjola, R., L. Newitt, D. Boteler, L. Trichtchenko, P. Fernberg, L. McKee,
D. Danskin, and G. Jansen van Beek, Modelling the disturbance caused by a dc-electrified railway to geomagnetic measurements, Earth Planets Space, 59, 943-949, 2007.

Schmucker, U., Anomalies of Geomagnetic variations in the Southwestern United States, University of California Press, Berkeley, 1970.

Soyer, W. and H. Brasse, Investigation of anomalous magnetic field variations in the central Andes of N Chile and SW Bolivia, Geophys. Res. Lett., 28(15), 3023-3026, 2001.

Varentsov, Iv. M. and EMTESZ WG, Joint robust inversion of quasi-2D MT/GDS data sets, in Protokol of the 21. kolloquium "Elektromagnetisch Tiefenforschung" Wohldenberg, edited by $\mathrm{O}$. Ritter, 111-123, http://bib.gfz-potsdam.de/emtf/2005/pdf/Varentsov_ EMTF_2005.pdf, 2005.

von Storch, H. and F. Zwiers, Statistical Analysis in Climate Research, p. 373, 496 pp, Cambridge University Press, 1999.

Wienert, K. A., Notes on geomagnetic observatory and survey practice, UNESCO Earth Sciences 5, UNESCO Paris, 1970.

C. Fox Maule (e-mail: cam@dmi.dk), P. Thejll, A. Neska, J. Matzka, L. W. Pedersen, and A. Nilsson 